\title{
Avaliação de Endoparasitoses gastrointestinais em ovinos em fazenda experimental na cidade de Porto Velho - RO, Brasil
}

\author{
Evaluation of gastrointestinal endoparasitosis in sheep in an experimental farm in the city of Porto \\ Velho - RO, Brazil \\ Evaluación de endoparasitosis gastrointestinal en ovinos en una granja experimental en la ciudad \\ de Porto Velho - RO, Brasil
}

Recebido: 27/07/2021 | Revisado: 03/08/2021 | Aceito: 12/10/2021 | Publicado: 14/10/2021

\author{
Thiago Vaz Lopes \\ ORCID: https://orcid.org/0000-0002-3192-1908 \\ Universidade Federal do Acre, Brasil \\ E-mail: thiagovlopes@ hotmail.com \\ João Gustavo da Silva Garcia de Souza \\ ORCID: https://orcid.org/0000-0002-5899-2490 \\ Centro Universitário Aparício Carvalho, Brasil \\ E-mail: joaogarciadesouza27@gmail.com \\ Josikelle Sabrinna Souza Santos \\ ORCID: https://orcid.org/0000-0003-4732-8970 \\ Centro Universitário Aparício Carvalho, Brasil \\ E-mail: josikelle.sabrinna@ hotmail.com \\ Maína Oliveira Agra \\ ORCID: https://orcid.org/0000-0003-3562-9901 \\ Centro Universitário Aparício Carvalho, Brasil \\ E-mail: aritgossubmissoes@gmail.com \\ Felipe Muriel Peixoto Rosas \\ ORCID: https://orcid.org/0000-0002-1876-2914 \\ Centro Universitário Aparício Carvalho, Brasil \\ E-mail: Felipe_muriel@hotmail.com \\ Thaís de Almeida Souza \\ ORCID: https://orcid.org/0000-0002-2722-9601 \\ Centro Universitário Aparício Carvalho, Brasil \\ E-mail: thaís_medvet@hotmail.com \\ Igor Mansur Muniz \\ ORCID: https://orcid.org/0000-0003-0863-6647 \\ Universidade Federal de Rondônia, Brasil \\ E-mail: igor.mansur@unir.br \\ Sandro de Vargas Schons \\ ORCID: https://orcid.org/0000-0001-9811-5356 \\ Universidade Federal de Rondônia, Brasi \\ E-mail: sandroschons@unir.br \\ Fernando Andrade Souza \\ ORCID: https://orcid.org/0000-0002-9474-9404 \\ Universidade Federal do Paraná, Brasil \\ E-mail: femedvet@yahoo.com.br
}

\begin{abstract}
Resumo
O parasitismo gastrointestinal é de suma importância na criação zootécnica de pequenos ruminantes. Dentre estas, se destacam nematóides do gênero Strongyloides, que proporciona uma das mais importantes alterações patológicas gastrintestinais de ruminantes de produção. Esta pesquisa tem por objetivo analisar a ocorrência de endoparasitos em ovinos que são vermifugados regularmente. O presente trabalho avaliou 30 animais de uma fazenda Experimental através da técnica de Willis e Mollay modificado. Conforme os resultados dos exames de fezes, pôde-se observar que apesar de terem sido vermifugados, a maioria dos animais estavam contaminados com Strongyloides spp., Haemonchus spp. e o Trichostrongylus spp. Portanto pode-se concluir que o anti-helmíntico utilizado não teve atividade totalmente eficaz sobre os helmintos.
\end{abstract}

Palavras-chave: Endoparasitose; Ruminantes; Strongyloides spp.; Antiparasitários; Trichostrongylus spp. 


\begin{abstract}
Gastrointestinal parasitism is of paramount importance in the animal husbandry of small ruminants. Among these, nematodes of the Strongyloides genus stand out, which provides one of the most important gastrointestinal pathological changes in production ruminants. This research aims to analyze the occurrence of endoparasites in sheep that are regularly dewormed. The present work evaluated 30 animals from an Experimental farm using the modified Willis and Mollay technique. According to the results of the feces exams, it could be observed that despite having been dewormed, most of the animals were contaminated with Strongyloides spp., Haemonchus spp. and Trichostrongylus spp. Therefore, it can be concluded that the anthelmintic used did not have a fully effective activity on helminths.
\end{abstract}

Keywords: Endoparasitosis; Ruminants; Strongyloides spp.; Antiparasitic; Trichostrongylus spp.

\title{
Resumen
}

El parasitismo gastrointestinal es de suma importancia en la cría de animales de pequeños rumiantes. Entre estos, se destacan los nematodos del género Strongyloides, que proporciona uno de los cambios patológicos gastrointestinales más importantes en la producción de rumiantes. Esta investigación tiene como objetivo analizar la aparición de endoparásitos en ovejas que son desparasitados regularmente. El presente trabajo evaluó 30 animales de una granja experimental utilizando la técnica modificada de Willis y Mollay. De acuerdo con los resultados de los exámenes de heces, se pudo observar que a pesar de haber sido desparasitados, la mayoría de los animales estaban contaminados con Strongyloides spp., Haemonchus spp. y Trichostrongylus spp. Por tanto, se puede concluir que el antihelmíntico utilizado no tuvo una actividad totalmente eficaz sobre los helmintos.

Palabras llave: Endoparasitosis; Rumiantes; Strongyloides spp.; Antiparasitario; Trichostrongylus spp.

\section{Introdução}

A ovinocultura tem ganhado destaque no agronegócio. Em 2016, o IBGE (2016) registrou mais de 18 milhões só no Brasil, sendo que em sua maioria com concentração na região nordeste do país.

A criação de ovinos vem se mostrado uma atividade com um rendimento satisfatório, pois proporciona ganhos financeiros tanto pela produção de carne, quanto à de leite, couro e lã. Além disso, essa atividade exige menor extensão de pastagem, quando comparado à bovina, suportando um número maior de animais/área (Gianlorenço, 2013). No entanto, uma das principais dificuldades no crescimento deste segmento são as parasitoses por nematódeos gastrintestinais, que sozinho representa o maior problema sanitário em pequenos ruminantes, chegando a ser um fator que inviabiliza economicamente a criação (Amarante \& Sales, 2007; Silva et al. 2019; Vieira, 2008; Vieira, 2005).

Dentre os endoparasitos gastrintestinais de ovinos, os mais encontrados fazem parte da família Trichostrongylidae (Ahid et al. 2008; Dias et al. 2018; Roberto et al. 2018a; Roberto et al. 2018b; Vieira, 2003). Nessa família, seus principais representantes são: Haemonchus contortus e Trichostrongylys axei, que parasitam o abomaso; e Trichostrongylus colubriformis e Cooperia sp., que habitam o intestino delgado; além de outras famílias e espécies que também acometem esses animais, como Strongyloides papillosus e Bunostomum trigonocephalum em intestino delgado e Oesophagostomum columbianum, Trichuris ovis e Trichuris globulosa, que e um endoparasitos de intestino grosso (Andriola et al., 2011; Costa et al., 2011; Fonseca et al., 2013). Estes parasitos são os maiores responsáveis por diminuir o desenvolvimento dos animais, principalmente dos mais jovens por conta de sua imunidade, além de aumentar a mortalidade dos mesmos (Yoshihara, 2012).

Segundo Amarante et al. (2004), para que se obtenha um maior sucesso no combate aos helmintos, é de grande necessário a realização de um controle embasado no conhecimento das espécies de nematódeos presentes nos animais da região, assim como a epidemiologia destes. Os mesmos autores ressaltaram que o sistema de manejo zootécnico associado aos estudos epidemiológicos dos helmintos pode resultar na diminuição na utilização de medicamentos nos animais. Outro problema adicional que esta atividade vem passando e referente o uso indiscriminado dessas drogas que resultou na seleção de cepas resistentes aos diferentes grupos químicos de anti-helmínticos existentes (Amarante et al. 1992; Minho, 2014; Vieira, 2003) e, consequentemente, estes produtos não vem realizando o controle das verminoses de maneira satisfatória.

Esta pesquisa tem por objetivo analisar a ocorrência de endoparasitos em ovinos que são vermifugados regularmente, pesquisado na Fazenda Experimental na cidade de Porto Velho, localizada no estado de Rondônia. 


\section{Material e Métodos}

Os ovinos da fazenda experimental na cidade de Porto Velho, no estado de Rondônia, foram avaliados clinicamente, através da anamnese de todos os 30 animais. Após isso, foi realizado a coleta das fezes antes e após a vermifugação dos animais, afim de realizar uma avaliação qualitativa de possíveis endoparasitos no plantel.

A coleta foi realizada entre Outubro e Novembro de 2019, no período da manhã. As amostras foram coletadas direto do reto do animal, com luvas de procedimento, para que evite contaminação com helmintos de vida livre do solo. Foram coletadas cerca de trinta amostras e colocadas em coletor universal, onde cada amostra teve em torno de $2 \mathrm{~g}$ a $4 \mathrm{~g}$, sendo identificada por animal e mantidas sob refrigeração até o momento do processamento no laboratório de Patologia Clínica de um Hospital veterinário de uma instituição de ensino superior da cidade.

A técnica utilizada foi a de Willis e Mollay (1921) modificado, onde foi homogeneizado em um Becker 2g de fezes com $20 \mathrm{ml}$ da solução hipersaturada de $\mathrm{NaCl}$, a solução foi filtrada com uma tamis, onde passou a suspensão filtrada para outro Becker menor, com quantidade suficiente para formar um menisco convexo. Então foi colocada uma lâmina de vidro sobre o Becker, procurando fazer com que a lâmina entre em contato com o líquido, evitando bolhas de ar entre a lâmina e o líquido, onde foi deixado repousar por 15 minutos. Após o tempo foi feito a leitura (contagem dos ovos) na lâmina, com o auxilio do microscópio, iniciado na objetiva de $4 \mathrm{x}$ e verificando todos os campos da lâmina. Os ovos encontrados foram calculados separadamente por gênero ou grupo de helmintos.

\section{Resultados e Discussão}

O controle dos parasitos é realizado com anti-helmínticos, visando reduzir os números da carga parasitológica dos animais e promover a descontaminação das pastagens (Charles, 1989)

A maioria dos animais deste trabalho pertence a categoria fêmeas, com um total de 20 amostras e 10 de machos.

A coleta dos animais antes de serem vermifugados foi realizada no dia nove de outubro. A administração de antihelmíntico (Ivermectina) foi no dia vinte e três de outubro e a segunda coleta realizada no dia vinte e nove de outubro de 2019.

Das trinta amostras analisadas antes da vermifugação, vinte e seis $(88,67 \%)$ animais apresentaram ovos presente nas fezes. Destas, dezesseis animais eram fêmeas, tendo uma porcentagem de 61,54\%, e dez machos, com uma porcentagem de $38,46 \%$ dos $88,67 \%$. Tudo isso quando submetidas ao método de flutuação de Willis-Mollay, como é mostrado na Tabela 1.

Tabela 1 - Parasitos detectados nas amostras fecais dos ovinos antes da vermifugação com Ivermectina, identificados por meio da técnica de flutuação.

\begin{tabular}{lc|c|r}
\hline \multicolumn{3}{c}{ Contagem antes da vermifugação } \\
\hline Gêneros de Parasitos & Machos & Fêmeas & \multicolumn{2}{c}{ Total } \\
\hline Strongyloides spp. & $10 / 10(100 \%)$ & $16 / 20(93,75 \%)$ & $26 / 30(88,67 \%)$ \\
Haemonchus spp. & $4 / 10(40 \%)$ & $3 / 20(18,75 \%)$ & $7 / 30(23,57 \%)$ \\
Trichostrongylus spp. & $7 / 10(70 \%)$ & $6 / 20(37,5 \%)$ & $13 / 30(43,33 \%)$ \\
\hline
\end{tabular}

Fonte: Autores.

Já nas amostras coletadas após a vermifugação dos animais foram encontrados ovos em vinte (66,67\%) amostras, sendo onze fêmeas $(55 \%)$ e nove machos $(45 \%)$, quando submetidas ao método de flutuação de Willis-Mollay, assim como demonstrado na Tabela 2. 
Tabela 2 - Parasitos detectados nas amostras fecais dos ovinos depois da vermifugação com Ivermectina, identificados por meio da técnica de flutuação.

\begin{tabular}{lc|c|c}
\hline \multicolumn{3}{c}{ Contagem depois da vermifugação } & \multicolumn{2}{c}{ Total } \\
\hline Gêneros de Parasitos & Machos & $11 / 20(55 \%)$ & $17 / 30(66,67 \%)$ \\
\hline Strongyloides spp. & $9 / 10(90 \%)$ & $2 / 20(18,18 \%)$ & $5 / 30(16,67 \%)$ \\
Haemonchus spp. & $3 / 10(33,33 \%)$ & & $9 / 30(30 \%)$ \\
Trichostrongylus spp. & $5 / 10(55,55 \%)$ & $4 / 20(36,36 \%)$ & \\
\hline
\end{tabular}

Fonte: Autores.

Através dos resultados dos exames coproparasitológicos pode-se observar que apesar de terem sido vermifugados, 56,67\% dos animais ainda estavam infectados por Strongyloides spp. Este resultado concorda com os dados de Amarante (2004) que verificou que Strongyloides spp, são uns dos principais parasitas de ovinos. O aparecimento de parasitas uma semana após a administração do anti-helmíntico mostrou que a droga utilizada não teve uma ação totalmente efetiva sobre os parasitas sugerindo uma possível resistência ao medicamento.

Segundo o que Andriola et al. (2011) relatam, nematóides da família Trichostrongylidae estão frequentemente relacionados às infecções parasitárias em rebanhos ovinos e os gêneros de maior importância dessa família são o Haemonchus spp. e o Trichostrongylus spp., corroborando com o que foi observado no presente trabalho, que constatou uma prevalência de $30 \%$ do gênero Trichostrongylus spp. em animais já everminados.

\section{Considerações Finais}

De acordo com os resultados obtidos pode-se concluir que o anti-helmíntico utilizado não teve atividade prolongada. A presença de parasitos uma semana após a vermifugação pode ser devido a uma possível resistência dos parasitos ao medicamento utilizado ou a grande infestação do meio ambiente por larvas infectantes.

\section{Referências}

Ahid, S. M. M. et al. (2008). Parasitos gastrintestinais em caprinos e ovinos da região oeste do Rio Grande do Norte, Brasil. Ciência Animal Brasileira, 9(1): $212-218$.

Amarante, A. F. T. et. al. (1992). Efeito da administração de oxfendazol, ivermectina e levamisol sobre os exames coproparasitológicos de ovinos. Braz. J Vet. Res. Anim. Sci, 29(1): 31-38.

Amarante, A. F. T. \& Sales, R. O. (2007). Controle de Endoparasitoses dos Ovinos: Uma Revisão. Revista Brasileira de higiene e Sanidade Animal, 1(2): 1436.

Amarante, A. F. T. et al. (2004). Resistance of santa ines, suffolk and ile de France lambs to naturally acquired gastrointestinal nematode infections. Veterinary Parasitology, 120(1-2): 91-106.

Andriola, L. C. et al. (2011). Diagnóstico de principais helmintos em caprinos no município de Grossos-RN. Revista Científica de Produção Animal, 13(1): $141-144$.

Costa, V. M. M. et al. (2011). Controle das parasitoses gastrintestinais em ovinos e caprinos na região semiárida do Nordeste do Brasil. Pesquisa Veterinária Brasileira, 31(1): 65-71.

Dias, G. F. et al. (2018). Parasitos gastrintestinais em ovinos de uma propriedade rural do estado do Piauí, PUBVET, 12(8): A146, 1-6.

Fonseca, Z. A. A. S. (2013). Helmintos gastrintestinais de caprinos leiteiros do Município de Afonso Bezerra, Rio Grande do Norte, Brasil. PUBVET, 7(19): Ed. 242, 1598 .

Gianlorenço, V. K. (2013). Produção de carne ovina pode ser mais rentável que bovina. São Paulo: SEBRAE. 
Research, Society and Development, v. 10, n. 13, e311101318603, 2021

(CC BY 4.0) | ISSN 2525-3409 | DOI: http://dx.doi.org/10.33448/rsd-v10i13.18603

IBGE, Instituto Brasileiro de Geografia e Estatística. 2016. Produção da Pecuária Municipal, 44: 1-53.

Jesus, A. R. et al. (2018). Controle de endoparasitas em ovinos recebendo frutos de nim (Azadirachta indica) no suplemento mineral. 55 ${ }^{a}$ Reunião anual da sociedade Brasileira de zootecnia $28^{\circ}$ Congresso Brasileiro de Zootecnia.

Minho, A. P. (2014). Endoparasitoses de ovinos: Conhecer para combater. Bagé: Embrapa Rio Grande do Sul. 19p.

Roberto, F. F. S. et al. (2018). Avaliação de resistência e susceptibilidade a nematódeos gastrintestinais em ovelhas a pasto. Boletim De Indústria Animal, 75(1): 44-51. https://doi.org/10.17523/bia.v75n1p44

Roberto, F. F. S. et al. (2018). Nematoides gastrintestinais na ovinocultura de corte sob regime de pastejo. PUBVET, 12(04): a65, 1-12.

Silva, M. F. S. et al. (2019). Endoparasitos como fator de importância na produção ovina. IV COLÓQIO ESTADUAL DE PESQUISA MULTIDISCIPLINAR II CONGRESSO NACIONAL DE PESQUISA MULTIDISCIPLINAR.

Vieira, L. S. (2003). Alternativas de controle da verminose gastrintestinal dos pequenos ruminantes (Circular técnica 29, 10 p.). Sobral, Ceará, Ministério da Agricultura, Pecuária e Abastecimento (MAPA).

Vieira, L. S. (2005). Endoparasitoses Gastrintestinais em Caprinos e Ovinos. Sobral: Embrapa Caprinos. 58p.

Vieira L. S. (2008). Métodos alternativos de controle de nematóides gastrintestinais em caprinos e ovinos. Revta Ciênc. Tecnol. Agropec. 2(2): 49-56.

Willis, H. H. (1921). A simple levitation method for the detection of wookworm ova. Medicine Journal of Australia, 8: 375-376.

Yoshihara, E. (2012). Avaliação de métodos alternativos no controle de nematódeos gastrintestinais em ovinos. Pesquisa \& Tecnologia, 9(2): 1-5. 